\title{
Precision measurement of vector and tensor analyzing powers in elastic deuteron-proton scattering
}

\author{
H. Mardanpour ${ }^{1, a}$, H.R. Amir-Ahmadi ${ }^{1}$, A. Deltuva ${ }^{2}$, K. Itoh ${ }^{3}$, N. Kalantar-Nayestanaki ${ }^{1}$, T. Kawabata ${ }^{4}$, H. \\ Kuboki $^{5}$, Y. Maeda ${ }^{4}$, J.G. Messchendorp ${ }^{1, b}$, S. Sakaguchi ${ }^{4}$, H. Sakai ${ }^{4,5}$, N. Sakamoto ${ }^{4}$, Y. Sasamoto ${ }^{4}$, M. Sasano $^{5}$, \\ K. Sekiguchi ${ }^{6}$, K. Suda ${ }^{4}$, Y. Takahashi ${ }^{5}$, T. Uesaka ${ }^{4}$, H. Witała ${ }^{7}$, and K. Yako ${ }^{5}$ \\ 1 Kernfysisch Versneller Instituut (KVI), University of Groningen, Zernikelaan 25, 9747 AA, Groningen, The Netherlands \\ 2 Centro de Fisica Nuclear da Universidade de Lisboa, P-1649-003 Lisboa, Portugal \\ 3 Department of Physics, Saitama University, Saitama 338-8570, Japan \\ 4 Center for Nuclear Study, University of Tokyo, Tokyo, Japan \\ 5 Department of Physics, University of Tokyo, Tokyo, Japan \\ 6 Nishina Center, RIKEN, Tokyo, Japan \\ 7 M. Smoluchowski Institute of Physics, Jagiellonian University, Kraków, Poland
}

Received: 8 November 2006 / Revised: 7 February 2007

Published online: 1 March 2007 - (C) Società Italiana di Fisica / Springer-Verlag 2007

Communicated by M. Garçon

\begin{abstract}
High-precision vector and tensor analyzing powers of elastic deuteron-proton $(\boldsymbol{d}+p)$ scattering have been measured at intermediate energies to investigate effects of three-nucleon forces. Angular distributions in the range of $70^{\circ}-120^{\circ}$ in the center-of mass frame for incident-deuteron energies $E_{d}^{l a b}=130$ and $180 \mathrm{MeV}$ were obtained using the RIKEN facility. The beam polarization was unambiguously determined by measuring the ${ }^{12} \mathrm{C}(\boldsymbol{d}, \alpha){ }^{10} \mathrm{~B}\left(2^{+}\right)$reaction at $0^{\circ}$. Results of the measurements are compared with stateof-the-art three-nucleon calculations. The present modeling of nucleon-nucleon forces and its extension to the three-nucleon system is not sufficient to describe the high-precision data consistently and requires, therefore, further investigation.
\end{abstract}

PACS. 21.30.-x Nuclear forces $-21.45 .+\mathrm{v}$ Few-body systems - 24.70.+s Polarization phenomena in reactions - 25.45.De Elastic and inelastic scattering

\section{Introduction}

The properties of nuclei and the dynamics of nuclear reactions are determined predominantly by the pair-wise nucleon-nucleon (NN) interaction [1]. The longest-range two-nucleon force (2NF) is due to the exchange of a pion, an idea that goes back to the work of Yukawa in 1935 [2, $3]$. At present, existing $2 \mathrm{NF}$ models provide an excellent description of the high-quality database of proton-proton and neutron-proton scattering and of the properties of the deuteron. For the simplest three-nucleon system, the triton, an exact solution of the three-nucleon Faddeev equations employing 2NFs clearly underestimates the experimental binding energy [4], and therefore shows that 2NFs are not sufficient to describe the three-nucleon system accurately. For heavier systems, the deviations between the calculated and the measured binding energies become even larger [5]. Deficiencies of theoretical predictions based on

\footnotetext{
a e-mail: mardanpur@kvi.nl

b e-mail: messchendorp@kvi.nl
}

pair-wise nucleon-nucleon potentials have been observed in three-nucleon scattering observables as well. For example, rigorous Faddeev calculations [6] solely based on modern NN interactions fail to describe high-precision differential cross-sections in proton-deuteron elastic scattering at intermediate energies obtained at KVI $[7,8]$ and at RIKEN [9]. A large part of the previously described discrepancies can be resolved by introducing additional three-nucleon forces (3NFs) [10].

Most of the present-day $3 \mathrm{NFs}$ are based on a refined version of the Fujita-Miyazawa force [11] in which a $2 \pi$ exchange mechanism is incorporated by an intermediate $\Delta$ excitation of one of the nucleons. Later, more refined ingredients have been added as in Urbana IX [12] and Tucson-Melbourne (TM') [13] allowing for additional processes contributing to the rescattering of the mesons on an intermediate excited nucleon. A different approach is provided by the Hannover theory group, where the $\Delta$-isobar is treated on the same basis as the nucleon, resulting in a coupled-channel potential CD-Bonn $+\Delta$ [14] with pair-wise nucleon-nucleon and nucleon- $\Delta$ interactions me- 
diated through the exchange of $\pi, \rho, \omega$, and $\sigma$ mesons. Within this self-consistent framework, the $\Delta$-isobar excitation mediates an effective $3 \mathrm{NF}$ with prominent FujitaMiyazawa and Illinois ring-type contributions [5].

A measurement of differential scattering cross-sections in three-nucleon systems is one of the tools used to study the general nature of the 3NF. More detailed information can be obtained by measuring other observables such as analyzing powers. For instance, the spin-dependent part of the $3 \mathrm{NF}$ can be studied specifically by measuring the vector and tensor analyzing powers using polarized proton and deuteron beams. Precision measurements of the vector analyzing power of the proton in elastic protondeuteron scattering have been performed at various beam energies ranging from 90 to $250 \mathrm{MeV}[8,15-17]$. Data on vector and tensor analyzing powers in $\boldsymbol{d}+p$ scattering are, however, scarce. Vector and tensor analyzing powers at various energies of $75-187$ [18], 191, and $395 \mathrm{MeV}$ [19] have been obtained in the past. Most of these data do not have sufficient precision and, therefore, not enough sensitivity to study $3 \mathrm{NF}$ effects. The only precision data covering a large angular range were obtained using a polarized deuteron beam with energies of 140,200 and $270 \mathrm{MeV}$ [9, $20]$. For a systematic study of the $3 \mathrm{NF}$, a more extensive database with higher-precision data is essential.

This paper describes a precision measurement of vector and tensor analyzing powers in $\boldsymbol{d}+p$ elastic scattering. The experiment was conducted at the RIKEN accelerator research facility using a polarized deuteron beam with energies of 130 and $180 \mathrm{MeV}$. The paper continues with a description of the experimental facility and the techniques used to measure the spin observables. The results will be compared and interpreted using modern Faddeev calculations as described earlier. The paper ends with a summary and conclusions.

\section{Experimental procedure and results}

Vector and tensor analyzing powers in $\boldsymbol{d}+p$ elastic scattering at $E_{d}^{l a b}=130$ and $180 \mathrm{MeV}$ were measured at the RIKEN accelerator research facility. The experiment was composed of two setups. In the first setup, the polarization of the deuteron beam was determined by measuring the ${ }^{12} \mathrm{C}(\boldsymbol{d}, \alpha){ }^{10} \mathrm{~B}\left(2^{+}\right)$reaction at $0^{\circ}$ using the SMART spectrograph. In the second setup, asymmetries in elastic $\boldsymbol{d}+p$ scattering were measured simultaneously with the SMART measurements using the D-room in-beam polarimeter. The vector and tensor analyzing powers in $\boldsymbol{d}+p$ elastic scattering were subsequently obtained by combining the results from the first and second setups. A detailed description of the experimental method can be found in ref. [21]. In this paper, we only provide a brief summary of the procedure.

The vector and tensor polarized deuteron beams were provided by the atomic beam polarized ion source [22]. In this experiment, the polarization states of the deuteron beam were switched between off-mode $\left(p_{Z}, p_{Z Z}\right)=$ $(0,0)$, up-mode $\left(p_{Z}, p_{Z Z}\right)=(1 / 3,+1)$, and down-mode $\left(p_{Z}, p_{Z Z}\right)=(1 / 3,-1)$. The values between the brackets correspond to the maximum theoretical values of the vector $\left(p_{Z}\right)$ and tensor $\left(p_{Z Z}\right)$ polarizations. The three different polarization states were switched every $5 \mathrm{~s}$ by changing the RF transition fields of the polarized ion source. The experimental polarization values were typically $60-80 \%$ of the maximum possible theoretical value. The beam intensity used during the experiment was typically $30 \mathrm{nA}$.

For the unambiguous determination of the beam polarization, we have exploited the relation between beam polarization and the ${ }^{12} \mathrm{C}(\boldsymbol{d}, \alpha){ }^{10} \mathrm{~B}\left(2^{+}\right)$reaction cross-section at $0^{\circ}$. Following the Madison convention [23], the crosssection of a scattered particle, $\sigma$, for a spin- 1 beam impinging on an unpolarized target and with an angle $\beta$ between the spin direction and the beam direction $(\hat{z})$ and an azimuthal angle $\phi$ defined to be $0^{\circ}$ for scattering to the left of the beam in the horizontal direction $(\hat{x})$ and $90^{\circ}$ for scattering downward $(-\hat{y})$, can be written as:

$$
\begin{aligned}
\sigma=\sigma_{0}[1 & +\sqrt{3} i T_{11}(\theta) p_{Z} \cos \phi \sin \beta \\
& +\frac{T_{20}(\theta) p_{Z Z}}{\sqrt{8}}\left(3 \cos ^{2} \beta-1\right) \\
& +\sqrt{3} T_{21} p_{Z Z} \cos \beta \sin \beta \sin \phi \\
& \left.-\frac{\sqrt{3}}{2} T_{22}(\theta) p_{Z Z} \cos 2 \phi \sin ^{2} \beta\right],
\end{aligned}
$$

where $i T_{11}, T_{20}, T_{22}$, and $T_{21}$ are the vector and various tensor analyzing powers, respectively, and $\theta$ is the polar scattering angle. The vector and tensor polarization of the beam are denoted as $p_{Z}$ and $p_{Z Z}$, respectively. The reaction cross-section for an unpolarized beam is given by $\sigma_{0}$.

For scattering at $\theta_{\text {c.m. }}=0^{\circ}$, the cross-section relation can be simplified, since $i T_{11}\left(0^{\circ}\right)=T_{22}\left(0^{\circ}\right)=T_{21}\left(0^{\circ}\right)=0$. In this case, eq. (1) becomes,

$$
\frac{\sigma}{\sigma_{0}}=1+\frac{T_{20}(\theta) p_{Z Z}}{\sqrt{8}}\left(3 \cos ^{2} \beta-1\right) .
$$

Equation (2) allows us to determine the tensor polarization of the beam by measuring the ratio between the polarized and unpolarized cross-sections at $0^{\circ}$. As a prerequisite, the angle $\beta$ should be known during the experiment. The angle, $\beta$, of the beam polarization can be manipulated at the ion source. The resulting polarization is preserved by the single turn extraction of the beam. In addition, the tensor analyzing power, $T_{20}$, should be known exactly and preferably at its maximum value. The latter is achieved by using the reaction ${ }^{12} \mathrm{C}(\boldsymbol{d}, \alpha)^{10} \mathrm{~B}\left(2^{+}\right)$as a measure for the beam polarization $[24,25]$. For symmetry reasons, this reaction has a maximum analyzing power of $T_{20}=1 / \sqrt{2}$ at $\theta_{c . m .}=0^{\circ}$. In addition, the $2^{+}$excited state can be identified uniquely by measuring the scattered $\alpha$ particle using the SMART spectrograph. In the ratio $\sigma / \sigma_{0}$ in eq. (2), detector-related parameters, such as solid angle and efficiencies, cancel out since the same detector is used for the different polarization states.

The SMART magnetic spectrograph [26] consists of a beam swinger and a cascade magnetic analyzer with two 


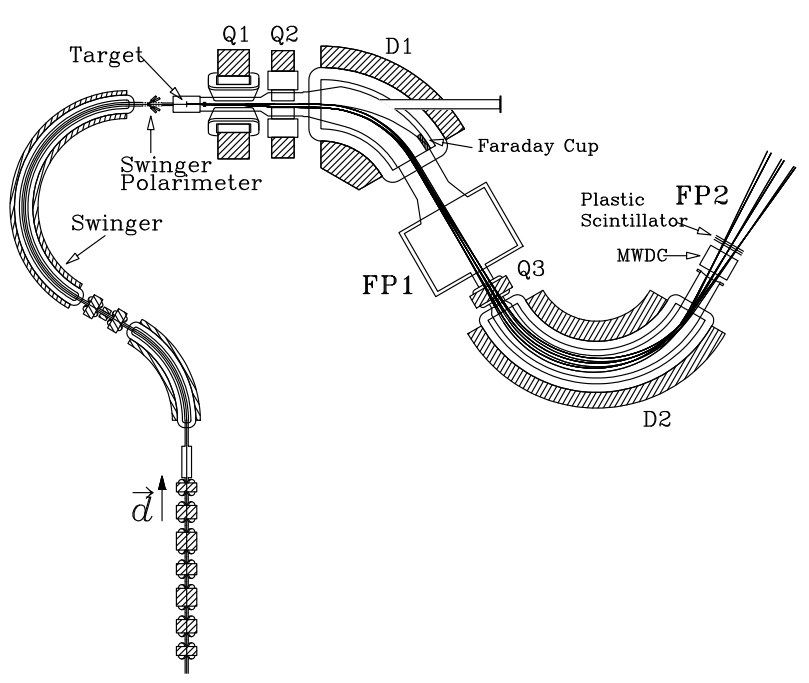

Fig. 1. A schematic top view of the SMART spectrograph which was used to measure the ${ }^{12} \mathrm{C}(\boldsymbol{d}, \alpha){ }^{10} \mathrm{~B}\left(2^{+}\right)$reaction at $0^{\circ}$.

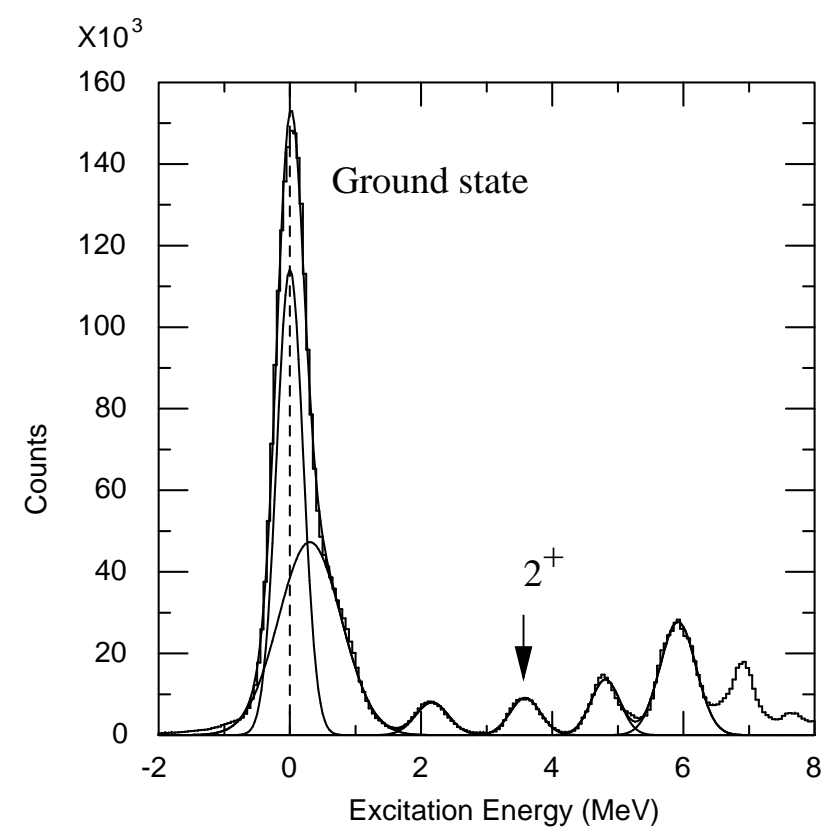

Fig. 2. The excited states of ${ }^{10} \mathrm{~B}$, obtained by a momentum analysis of the $\alpha$ particles in the reaction ${ }^{12} \mathrm{C}(\boldsymbol{d}, \alpha){ }^{10} \mathrm{~B}\left(2^{+}\right)$. The events stemming from the $2^{+}$state have been used to determine the beam polarization as described in the text.

focal planes. The three quadruples and two dipole magnets were set in a QQDQD configuration. The scattered $\alpha$ particles were momentum analyzed by the magnetic spectrograph and detected by a multiwire drift chamber and three plastic scintillators placed at the second focal plane. A schematic top view of the SMART spectrograph is given in fig. 1. In this experiment the scattering of the polarized deuteron beam from a ${ }^{12} \mathrm{C}$ target with a thickness of $20 \mathrm{mg} / \mathrm{cm}^{2}$ was studied with the SMART spectrograph.

Figure 2 shows the excitation spectrum of ${ }^{10} \mathrm{~B}$ obtained by a momentum analysis of the scattered $\alpha$ par-

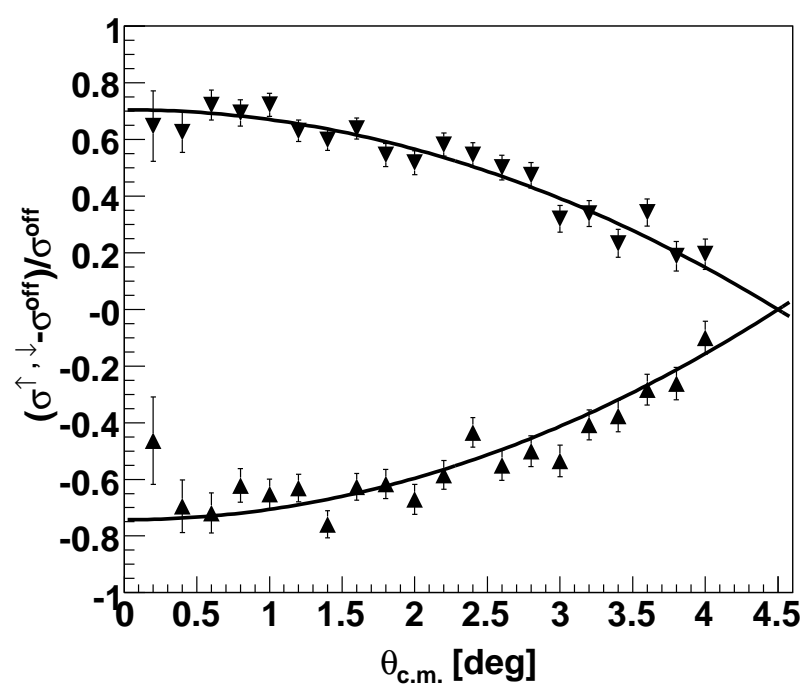

Fig. 3. Polarization asymmetry of the ${ }^{12} \mathrm{C}(\boldsymbol{d}, \alpha){ }^{10} \mathrm{~B}\left(2^{+}\right)$reaction as a function of the scattering angle in the center-of-mass frame. The two data sets were obtained from two polarization states of $\left(p_{Z}, p_{Z Z}\right)=(1 / 3,+1)$ and $\left(p_{Z}, p_{Z Z}\right)=(1 / 3,-1)$ normalized to the off polarization state $\left(p_{Z}, p_{Z Z}\right)=(0,0)$. The asymmetry at $\theta_{c . m}=0^{\circ}$ is determined by an extrapolation of asymmetries measured up to $4^{\circ}$ via a second-order polynomial fit.

ticles. A clear signal from the $2^{+}$state can be identified. These events are used for the extraction of the tensor polarization of the deuteron beam according to eq. (2). The polarization asymmetries, $\left(\sigma^{\uparrow}-\sigma^{\text {off }}\right) / \sigma^{\text {off }}$ and $\left(\sigma^{\downarrow}-\sigma^{o f f}\right) / \sigma^{o f f}$, of the $(d, \alpha)$ reaction were obtained. The cross-sections, $\sigma^{\uparrow}, \sigma^{\downarrow}$, and $\sigma^{o f f}$, correspond to a measurement with a beam polarization of $\left(p_{Z}, p_{Z Z}\right)=$ $(1 / 3,+1),\left(p_{Z}, p_{Z Z}\right)=(1 / 3,-1)$, and $\left(p_{Z}, p_{Z Z}\right)=(0,0)$, respectively. Note that we used $\sigma^{o f f}$ instead of $\sigma_{0}$. In an ideal case, $\sigma^{o f f}$ corresponds to the cross-section for a theoretically unpolarized beam. In our data analysis, however, a correction due to a small non-zero polarization in the $\left(p_{Z}, p_{Z Z}\right)=(0,0)$ mode was taken into account. A typical angular distribution of the asymmetries of the abovementioned reaction for two different polarization states is shown in fig. 3 . To obtain a precise asymmetry at $0^{\circ}$, a second-order polynomial was fitted to the observed angular distribution as shown by the solid lines. Fluctuations in the beam polarization were monitored by measuring these asymmetries in 20 time slices of 60 minutes each. These fluctuations were found to be small.

The D-room polarimeter at RIKEN was used to measure polarization asymmetries in the elastic $\boldsymbol{d}+p$ reaction. Combined with a simultaneous measurement of the beam polarization using the SMART spectrometer, vector and tensor analyzing powers in elastic $\boldsymbol{d}+p$ scattering are obtained. The D-room polarimeter consists of $4 \times 7$ plastic scintillators placed to the right $\left(\phi=0^{\circ}\right)$, left $\left(\phi=180^{\circ}\right)$, up $\left(\phi=90^{\circ}\right)$, and down $\left(\phi=270^{\circ}\right)$ of a solid $\mathrm{CH}_{2}$ target. The elastic $\boldsymbol{d}+p$ reaction was identified nearly background-free by a left-right or up-down coincidence requirement. For this, four scintillators were placed 


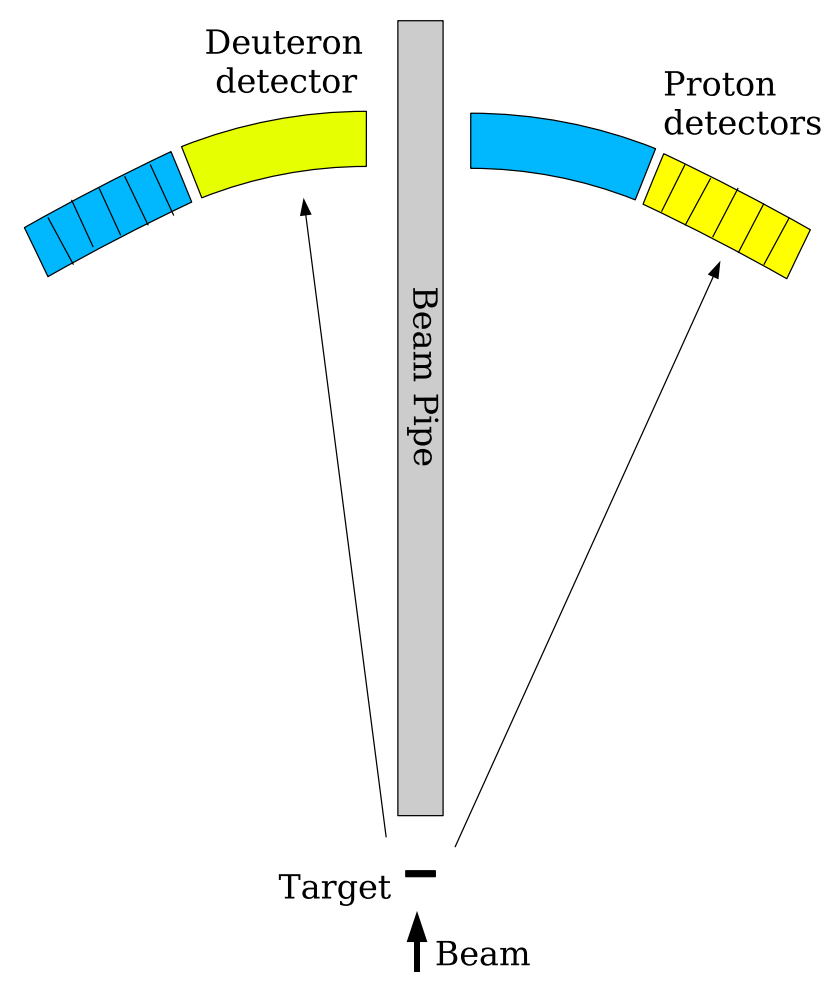

Fig. 4. A sketch of the experimental setup used to measure the elastic deuteron-proton scattering process. Six small proton detectors are placed in coincidence with one deuteron detector placed on the other side of the beam. The coincidence setup in light gray (yellow on-line) and in dark gray (blue on-line) correspond to an azimuthal plane of $\phi=0^{\circ}$ (left) and $\phi=180^{\circ}$ (right). The coincidence planes at $\phi=90^{\circ}$ and $270^{\circ}$ are not shown.

at a polar scattering angle of $25^{\circ}$ covering a large part of deuteron phase space. These detectors were required to be in coincidence with one of the remaining smaller proton detectors placed on the other side of the beam at polar scattering angles between 30 and 55 degrees. Figure 4 illustrates schematically the coincidence setup for the left and right side on the horizontal plane. The vertical plane is not shown in this picture.

Figure 5 shows the spectrum corresponding to the deposited energy of one of the proton detectors in coincidence with the opposite deuteron detector. This proton detector was placed at an angle of $30^{\circ}$, and it had a thickness of $10 \mathrm{~mm}$. All impinging protons punch through the detector and deposit an energy of about $15 \mathrm{MeV}$. The small tail on the left of the peak stems predominantly from hadronic interactions with the scintillator material. Furthermore, note that the pile-up background is negligible, which is indicated by the lack of events on the right side of the main peak. For the asymmetry determination in $\boldsymbol{d}+p$ scattering, events were selected within the gate shown in fig. 5 .

The vector and tensor analyzing powers can be obtained by making use of the $\phi$-dependence according to eq. (1). The analyzing powers have been obtained by a global $\chi^{2}$-minimization fit of all data from the SMART

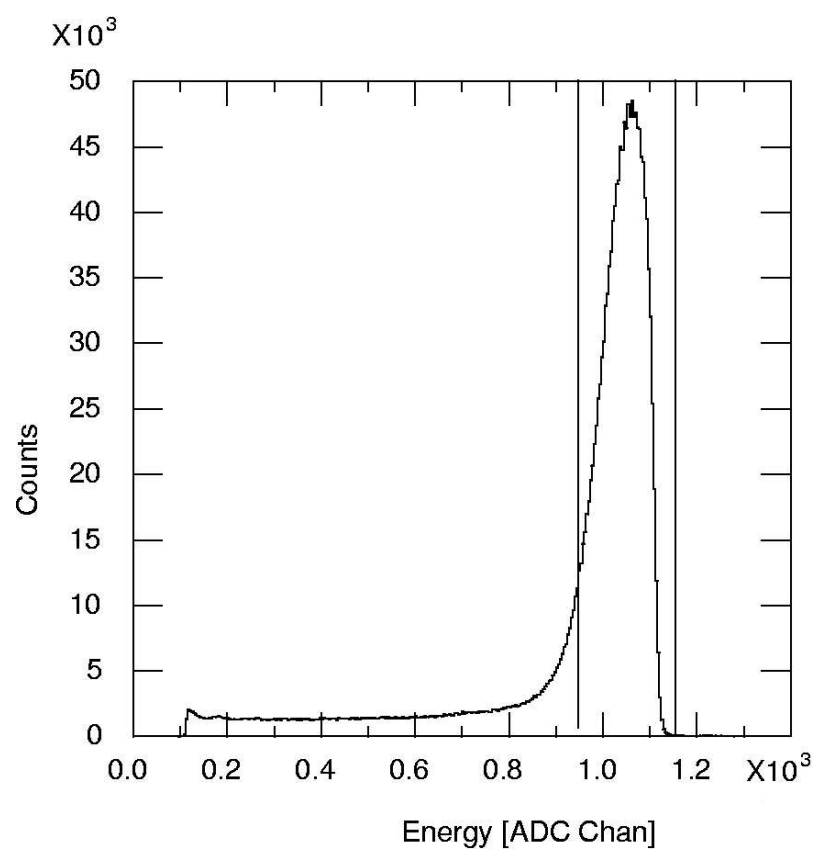

Fig. 5. The response of one of the proton detectors placed at $30^{\circ}$ and for a beam energy of $180 \mathrm{MeV}$. A clear signal stemming from the elastic deuteron-proton reaction can be observed with negligible background. The selected events used for the asymmetry measurement lie in the gate shown in the figure.

and the D-room polarimeter, using eq. (1). The free parameters in this fit were the analyzing powers, the tensor polarizations $p_{Z Z}^{\uparrow}, p_{Z Z}^{\downarrow}, p_{Z Z}^{o f f}$, and the cross-section for an unpolarized beam, $\sigma_{0}$. Note that as a consequence of the operation mode of the atomic polarized-beam source, the vector polarization is $1 / 3$ of the obtained tensor polarization, independent of the efficiencies of the transition units. The combined $\chi^{2}$ fitting procedure with 3 degrees of freedom was used to extract the experimental parameters and monitor them over time to estimate the systematic uncertainty.

With the geometry shown in fig. 4, the vector and tensor analyzing powers in elastic $\boldsymbol{d}+p$ scattering were measured for $\theta_{c . m} .=70^{\circ}-120^{\circ}$ at $E_{d}=130 \mathrm{MeV}$ and $\theta_{c . m .}=76^{\circ}-120^{\circ}$ at $E_{d}=180 \mathrm{MeV}$. The results for $E_{d}^{i a b}=130 \mathrm{MeV}$ are shown in fig. 6 and listed in table 1. Similarly, the results for $E_{d}^{l a b}=180 \mathrm{MeV}$ can be found in fig. 7 and in table 2. The error bars in figs. 6 and 7 indicate only statistical uncertainties together with a small point-to-point (PTP) uncertainty. The small PTP error is added to account for small, non-statistical, fluctuations of the data points as judged by comparison to a fit using a 4 th-order polynomial. These PTP uncertainties are added quadratically to the statistical uncertainties.

The dominant systematic uncertainty stems from the knowledge of the polar, $\beta$, and azimuthal, $\alpha$, angles of the polarization direction with respect to the beam direction. These angles were determined in a separate measurement which was conducted immediately after the $(\boldsymbol{d}, \alpha)$ and $\boldsymbol{d}+p$ measurements. In this measurement, the polarization axis precesses in the swinger dipole magnet of the 


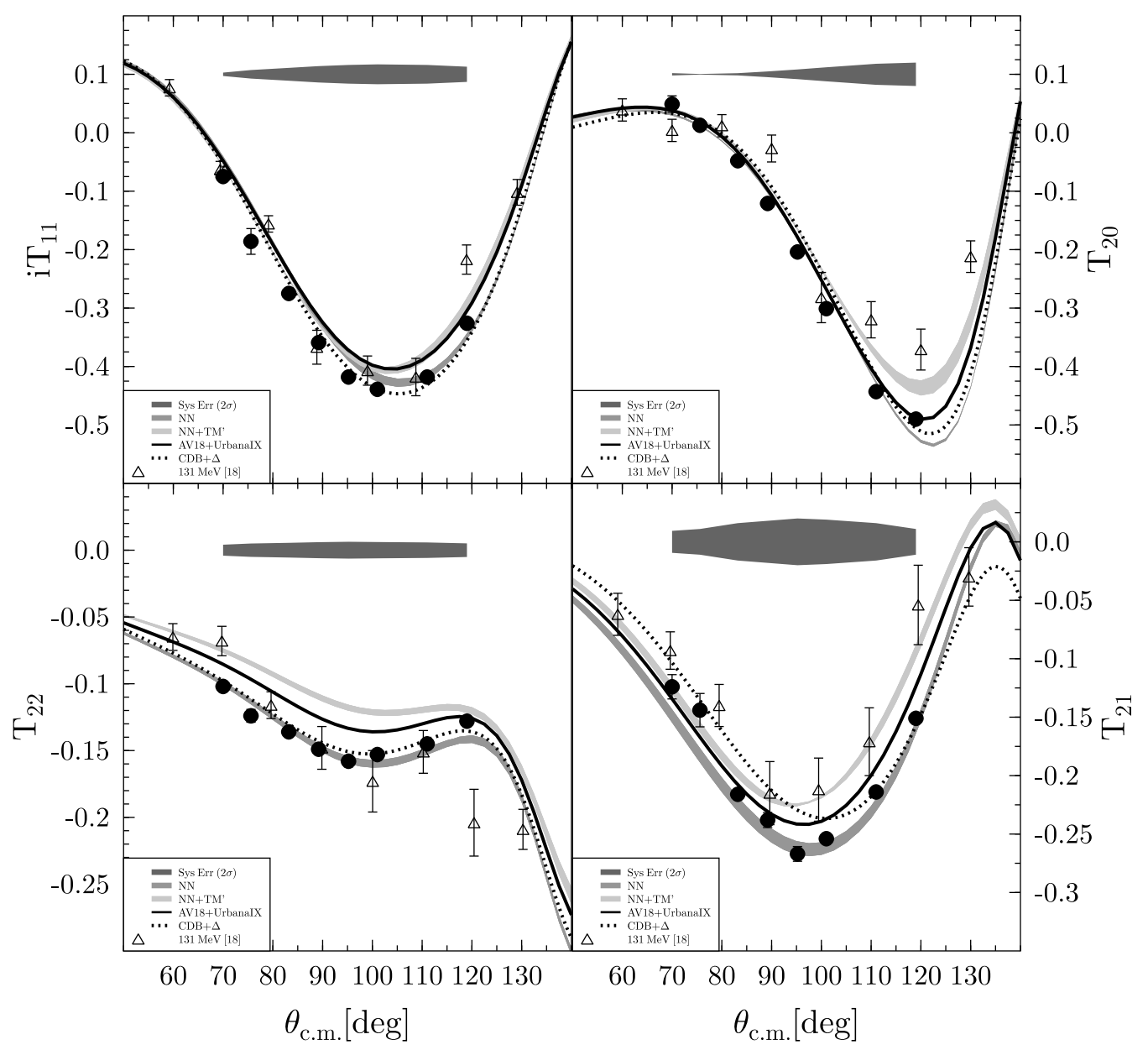

Fig. 6. Vector and tensor analyzing powers of the elastic $\boldsymbol{d}+p$ scattering at $E_{d}^{l a b}=130 \mathrm{MeV}$ as a function of $\theta_{c . m}^{\circ}$. Error bars are statistical plus a point-to-point uncertainty added in quadrature. In each panel, filled circles are data from the present experiment and open triangles are data from ref. [18] at $E_{d}^{l a b}=131 \mathrm{MeV}$. The dark-gray bands at the top of the panels represent the systematical uncertainty $(2 \sigma)$ for every data point. The other dark-gray bands correspond to calculations including only twonucleon potentials. The light-gray bands represent calculations including an additional Tucson-Melbourne TM' three-nucleon force as well. The solid lines correspond to results of a Faddeev calculation using the AV18 two-nucleon potential combined with the Urbana-IX (UIX) three-nucleon potential. The dotted line represents the results of a coupled-channel potential CDB $+\Delta$ calculations.

Table 1. Analyzing powers for elastic $\boldsymbol{d}+p$ scattering at $E_{d}^{l a b}=130 \mathrm{MeV}$ for different scattering angles in the center-of-mass frame. Statistical errors are quoted with the superscript st. and systematical errors with the superscript sys. Statistical errors are the output of a $\chi^{2}$ fitting procedure plus an additional point-to-point uncertainty added quadratically. The uncertainty in $\theta_{c . m}$. is $\pm 0.5^{\circ}$. Note that all the analyzing power values and errors have been multiplied by a factor 1000 .

\begin{tabular}{ccccccccccccc}
\hline$\theta_{c . m .}\left[^{\circ}\right]$ & $i T_{11}$ & $\Delta i T_{11}^{s t .}$ & $\Delta i T_{11}^{s y s .}$ & $T_{22}$ & $\Delta T_{22}^{s t .}$ & $\Delta T_{22}^{\text {sys. }}$ & $T_{20}$ & $\Delta T_{20}^{\text {st. }}$ & $\Delta T_{20}^{\text {sys. }}$ & $T_{21}$ & $\Delta T_{21}^{\text {st. }}$ & $\Delta T_{21}^{\text {sys. }}$ \\
\hline 70 & -75 & 6 & 3 & -102 & 3 & 4 & 49 & 14 & 2 & -124 & 10 & 9 \\
76 & -186 & 22 & 7 & -124 & 5 & 5 & 13 & 4 & 1 & -144 & 14 & 11 \\
83 & -275 & 4 & 11 & -136 & 2 & 6 & -48 & 4 & 2 & -216 & 4 & 16 \\
89 & -359 & 3 & 14 & -149 & 1 & 6 & -121 & 5 & 5 & -238 & 6 & 18 \\
95 & -418 & 4 & 16 & -158 & 4 & 6 & -204 & 6 & 8 & -267 & 6 & 20 \\
101 & -439 & 3 & 17 & -153 & 1 & 6 & -301 & 5 & 12 & -254 & 4 & 19 \\
111 & -418 & 3 & 16 & -145 & 1 & 6 & -443 & 5 & 18 & -214 & 4 & 16 \\
119 & -326 & 3 & 13 & -128 & 2 & 5 & -490 & 6 & 20 & -151 & 3 & 11 \\
\hline
\end{tabular}




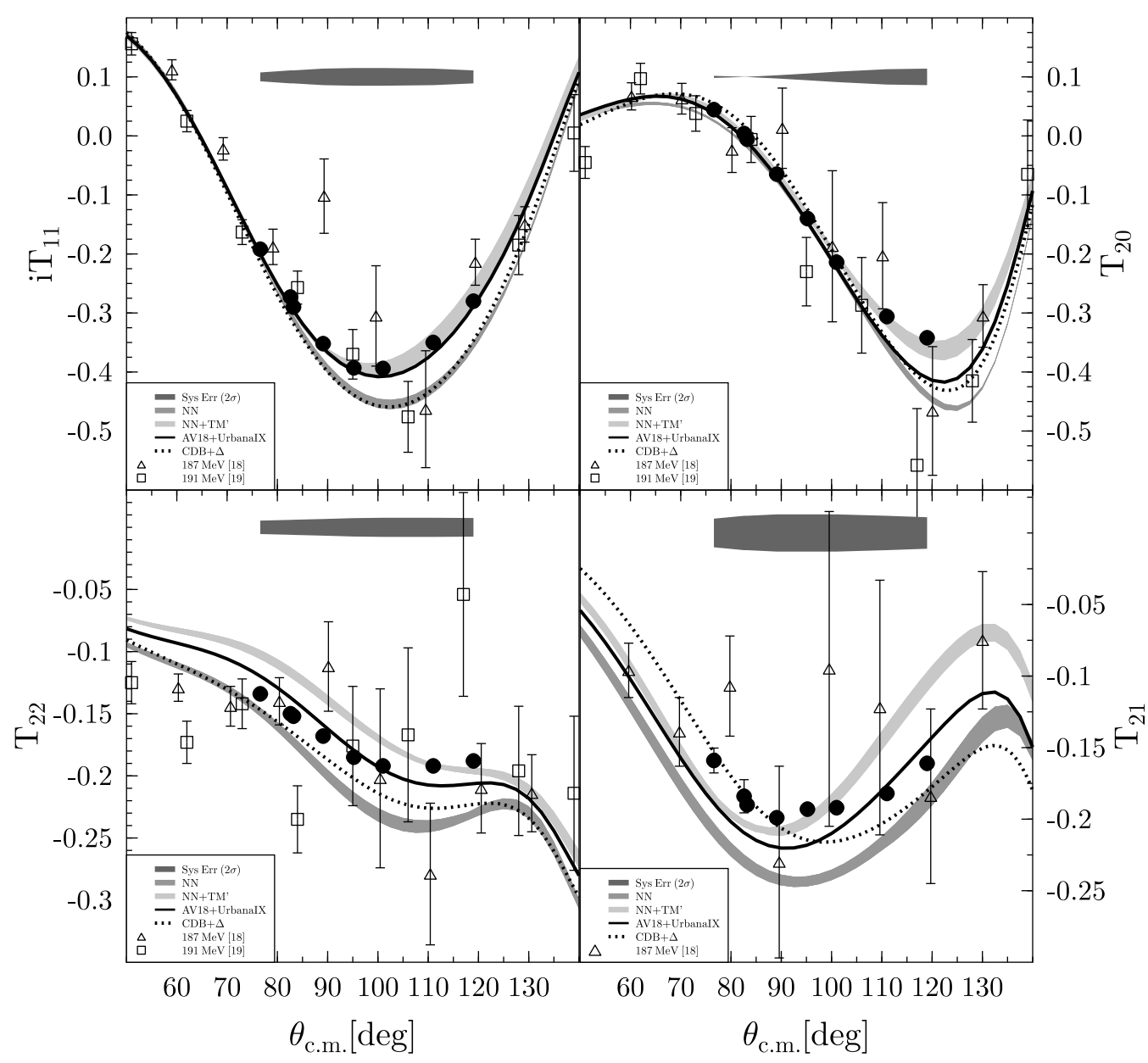

Fig. 7. Vector and tensor analyzing powers in the elastic $\boldsymbol{d}+p$ scattering at an incident deuteron beam energy of $E_{d}^{l a b}=180 \mathrm{MeV}$. The open triangles are data from ref. [18] and the open squares are data from ref. [19]. For a description of the bands and lines refer to fig. 6 .

Table 2. Analyzing powers for elastic $\boldsymbol{d}+p$ scattering at $E_{d}^{l a b}=180 \mathrm{MeV}$. See table 1 for more details.

\begin{tabular}{ccccccccccccc}
\hline$\theta_{c . m .}\left[^{\circ}\right]$ & $i T_{11}$ & $\Delta i T_{11}^{s t .}$ & $\Delta i T_{11}^{s y s .}$ & $T_{22}$ & $\Delta T_{22}^{s t .}$ & $\Delta T_{22}^{s y s .}$ & $T_{20}$ & $\Delta T_{20}^{s t .}$ & $\Delta T_{20}^{s y s .}$ & $T_{21}$ & $\Delta T_{21}^{\text {st. }}$ & $\Delta T_{21}^{\text {sys. }}$ \\
\hline 77 & -192 & 7 & 8 & -134 & 3 & 5 & 45 & 7 & 2 & -159 & 9 & 10 \\
83 & -273 & 9 & 11 & -150 & 4 & 6 & 4 & 8 & 1 & -184 & 12 & 12 \\
84 & -290 & 2 & 11 & -152 & 1 & 6 & -6 & 1 & 1 & -190 & 3 & 12 \\
89 & -352 & 3 & 14 & -168 & 1 & 7 & -65 & 1 & 3 & -199 & 3 & 13 \\
95 & -393 & 3 & 15 & -185 & 1 & 7 & -140 & 2 & 6 & -193 & 3 & 13 \\
101 & -394 & 3 & 15 & -192 & 1 & 8 & -214 & 3 & 9 & -192 & 3 & 13 \\
111 & -350 & 3 & 14 & -192 & 2 & 8 & -306 & 3 & 13 & -182 & 7 & 12 \\
119 & -280 & 3 & 11 & -188 & 2 & 8 & -342 & 4 & 14 & -161 & 4 & 11 \\
\hline
\end{tabular}

SMART spectrograph which was rotated by $90^{\circ}$ in a vertical plane. The precession angle is measured before and after the spin precession by using two polarimeters. For a beam energy of $130 \mathrm{MeV}$, the values $\beta=82.0^{\circ} \pm 0.5^{\circ}$ and $\alpha=2.0^{\circ} \pm 0.5^{\circ}$ were obtained, whereas $\beta=99.4^{\circ} \pm 0.5^{\circ}$ and $\alpha=2.0^{\circ} \pm 0.5^{\circ}$ were obtained for a deuteron beam energy of $180 \mathrm{MeV}$. Since the sensitivity to the $T_{21}$ analyzing power in eq. (1) goes as $\cos \beta \sin \beta$, as $\beta$ approaches $90^{\circ}$, the uncertainty in $T_{21}$ increases. As a consequence, the large systematic errors in $T_{21}$ are associated with the value of $\beta$ in these experiments. In addition, variations in the beam polarization angles during the experiment have been monitored by dividing the data into several bins in time, and were found to be small. Yet these non-statistical fluctuations were taken into account in our $\chi^{2}$ fitting procedure. The total systematic error is the quadratic sum of 


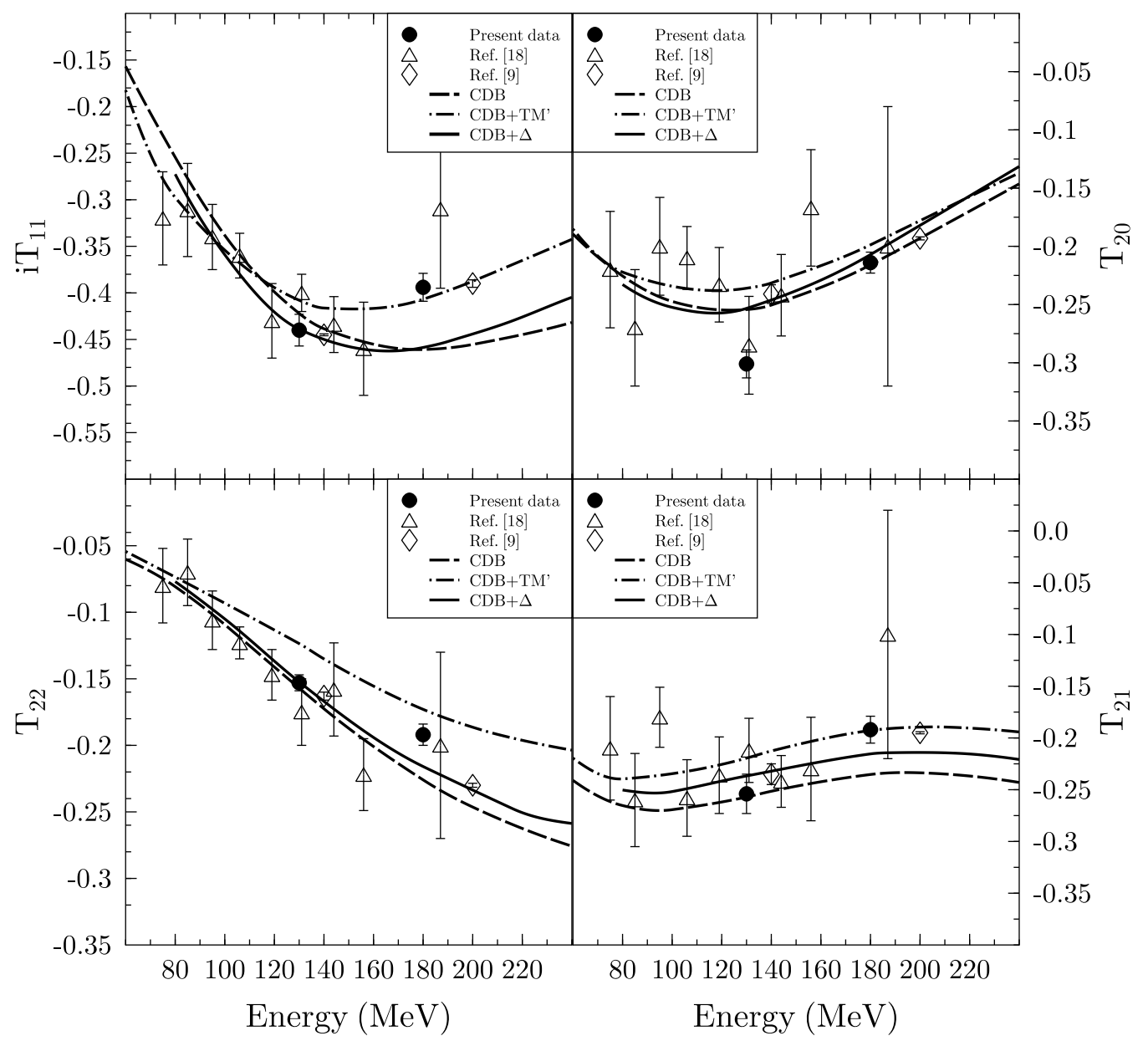

Fig. 8. A comparison of our measurements of vector and tensor analyzing powers in deuteron-proton elastic scattering with the world database at intermediate energies as a function of the incident beam energy. All data points correspond to a center-of-mass angle of $\theta_{c . m .}=100^{\circ}$. Our data are presented as filled circles, together with that of ref. [9] (open diamond) and of ref. [18] (open triangles). The predictions for $\mathrm{CDB}, \mathrm{CDB}+\mathrm{TM}$ ', and $\mathrm{CDB}+\Delta$ are represented by dashed, dash-dotted and solid lines, respectively.

these partial systematic errors which are given in tables 1 and 2 with superscript sys and are plotted as gray bands on top of the figures.

\section{Comparison between data and theory}

The measured vector and tensor analyzing powers are shown in figs. 6 and 7 by filled circles and compared to rigorous Faddeev calculations, represented by bands and lines. The dark-gray bands correspond to calculations including only two-nucleon potentials, namely Argonne V18 (AV18) [27], Charge-Dependent Bonn (CDB) [28], Nijmegen I (NIJM I), and Nijmegen II (NIJM II) [29]. The width of the bands indicates the variation in predictions for the three-body systems when using different twonucleon potentials. The light-gray bands represent calculations including an additional TM' three-nucleon force as well. The solid lines correspond to the results of a Faddeev calculation using the AV18 two-nucleon poten- tial combined with the Urbana-IX three-nucleon potential [12]. The dotted lines result from a calculation using the coupled-channel potential CDB $+\Delta[14]$. It should be noted that the calculations presented in this paper do not include the Coulomb interaction. The Hannover-Lisbon theory group, however, recently provided predictions including the Coulomb interaction $[30,31]$. For the polarization observables and energies presented here, its effect is found to be negligible, and therefore, has been left out to allow a fair comparison with the other predictions that do not include the Coulomb interaction. Also measurements from refs. $[18,19]$ at energies close to our energy are shown by open triangles and squares for every energy. They clearly show that the precision of the new measurements has been improved significantly.

A comparison between theory and experiment at $E_{d}=$ $130 \mathrm{MeV}$ shows that the Faddeev calculations based on a pure two-nucleon potential give a reasonable agreement with the measured polarization observables. At this energy, calculations with the inclusion of the well-established 
TM' three-nucleon force fail to describe the data, in particular for $T_{22}$. Calculations using the Urbana-IX threenucleon potential show, with the exception of $T_{20}$, significant discrepancies with the data as well. On the other hand, calculations using the coupled-channel potential, $\mathrm{CDB}+\Delta$, demonstrate that the polarization observables in the three-nucleon system can be described reasonably well by incorporating consistently an intermediate $\Delta$-resonance which seems to have a small effect for all observables except for $T_{21}$. Note that the discrepancies for $T_{21}$ can be partly ascribed to the large systematic uncertainties in this polarization observable.

At $E_{d}=180 \mathrm{MeV}$ the behavior of the Faddeev calculations with respect to the data changes drastically. The Faddeev calculations, incorporating two-nucleon forces only, show large deviations from the data for all observables. The inclusion of a TM' three-nucleon potential remedies these deficiencies significantly for $i T_{11}$ and $T_{20}$. However, the predictions for the tensor analyzing powers, $T_{22}$ and $T_{21}$, fail to describe the magnitude and the shape of the measured angular distributions. Furthermore, the model including the $\Delta$-resonance fails to describe a large part of these data as well, even though the same model gave a good description at $130 \mathrm{MeV}$.

From figs. 6 and 7 , we observe that the discrepancies between data and theory depend on the incident beam energy. As a further check on this, the beam-energy dependence of our data has been compared with the presently available world data base at intermediate energies. Results are shown in fig. 8. All data points correspond to a fixed angle of $\theta_{c . m .}=100 \pm 0.5^{\circ}$ in the center of mass. Our data (filled circles) are consistent with the data from ref. [18] (open triangles). In addition, the precision measurements of Sekiguchi et al. [9] at $E_{d}^{\text {lab }}=140$ and $200 \mathrm{MeV}$ are plotted as open diamonds. Results of the Faddeev calculations for the CDB potential without $3 \mathrm{NF}$ and with TM' and $\Delta$ are drawn as dashed lines, dash-dotted and solid lines, respectively.

The large deviations between our data and the rigorous predictions from Faddeev calculations based on modern two-nucleon potentials show that the observables shown in this paper are sensitive to $3 \mathrm{NF}$ effects. The role of the $\Delta$-resonance as a degree of freedom for $3 \mathrm{NF}$ has proved to be the dominant ingredient to describe well the vector analyzing powers in $\boldsymbol{p}+\boldsymbol{d}$ elastic scattering at intermediate energies from $108 \mathrm{MeV}$ to $190 \mathrm{MeV} /$ nucleon [8]. This paper, however, presents large discrepancies between data and a self-consistent coupled-channel model including a dynamic $\Delta$-resonance at an incident energy of $90 \mathrm{MeV} /$ nucleon for the vector and tensor analyzing powers of the deuteron. For these observables and energies, the role of the $\Delta$ as a $3 \mathrm{NF}$ is predicted to be small, whereas the data show that large $3 \mathrm{NF}$ effects are present. A description of $3 \mathrm{NF}$ effects using the phenomenological two-pion exchange approach such as the TM' $3 \mathrm{NF}$, which is added to a $2 \mathrm{NF}$, cannot remedy the observed discrepancies either. In addition to the models presented in this paper, other approaches are becoming available in the literature. One of these, namely chiral-perturbation theory, is based on the symmetries of quantum chromodynamics. Within this approach, nuclear forces are generated systematically $[32,33]$. Once higher orders are included in the calculations based on this theory for intermediate energies, one can make a reasonable comparison with the present data.

\section{Summary and conclusion}

The precision measurement of vector and tensor analyzing powers in elastic $\boldsymbol{d}+p$ scattering has been performed at intermediate energies of 130 and $180 \mathrm{MeV}$ in the centerof-mass angle range of $70^{\circ}-120^{\circ}$. Two different setups were used to measure the deuteron beam polarization via ${ }^{12} \mathrm{C}(\boldsymbol{d}, \alpha){ }^{10} \mathrm{~B}\left(2^{+}\right)$reaction and simultaneously the reaction asymmetries via elastic $\boldsymbol{d}+p$ reaction. Predictions of various $3 \mathrm{NF}$ models have been compared with the data in order to look for evidences of 3NF effects in this energy range. At $130 \mathrm{MeV}$, the agreement between data and calculations including only two-nucleon potentials is better for all observables. Also CDB $+\Delta$ describes the data better than calculations using other $3 \mathrm{NFs}$. At $180 \mathrm{MeV}$, the discrepancy between data and calculations is rather large. Calculations including TM' agree better for two analyzing powers of $i T_{11}$ and $T_{20}$ but the trend of the data for the other two analyzing powers, $T_{22}$ and $T_{21}$, matches none of the calculations. Our new data points are compared with the existing data base of deuteron analyzing powers in the energy range of 75-200 MeV. The energy dependence of all observables confirms that $3 \mathrm{NF}$ effects certainly exist but their exact nature should be investigated further.

We would like to thank the RIKEN accelerator group for doing an excellent job during this measurement and appreciate the RIKEN heavy-ion group for their hospitality and help during the experiment. Special thanks go to R.G.E. Timmermans, E. Stephan, St. Kistryn, A. Biegun, U. van Kolck, E.J. Stephenson, and C. Bailey for their valuable discussions and suggestions. This work was performed as part of the research program of the "Stichting voor Fundamenteel Onderzoek der Materie" (FOM) and was supported by the University of Groningen (RUG).

\section{References}

1. J. Carlson, R. Schiavilla, Rev. Mod. Phys. 70, 743 (1998).

2. H. Yukawa, Proc. Phys. Math. Soc. Jpn. 17, 48 (1935).

3. V. Stoks et al., Phys. Rev. C 47, 512 (1993); M.C.M. Rentmeester et al., Phys. Rev. Lett. 82, 4992 (1999).

4. A. Nogga et al., Phys. Rev. C 65, 054003 (2002).

5. S.C. Pieper et al., Phys. Rev. C 64, 014001 (2001).

6. W. Glöckle et al., Phys. Rep. 274, 107 (1996).

7. K. Ermisch et al., Phys. Rev. C 68, 051004(R) (2003).

8. K. Ermisch et al., Phys. Rev. C 71, 064004 (2005).

9. K. Sekiguchi et al., Phys. Rev. C 65, 034003 (2002).

10. H. Witała et al., Phys. Rev. Lett. 81, 1183 (1998).

11. J. Fujita, H. Miyazawa, Prog. Theor. Phys. 17, 360 (1957).

12. B.S. Pudliner et al., Phys. Rev. C 56, 1720 (1997).

13. S.A. Coon et al., Few-Body Syst. 30, 131 (2001); J.L. Friar et al., Phys. Rev. C 54, 53 (1999). 
14. A. Deltuva et al., Phys. Rev. C 68, 024005 (2003).

15. R. Bieber et al., Phys. Rev. Lett. 84, 606 (2000).

16. K. Ermisch et al., Phys. Rev. Lett. 86, 5862 (2001).

17. K. Hatanaka et al., Phys. Rev. C 66, 044002 (2002).

18. H. Witała et al., Few-Body Syst. 15, 67 (1993); J.M. Cameron et al., Nucl. Instrum. Methods A 305, 257 (1991).

19. M. Garçon et al., Nucl. Phys. A 458, 287 (1986).

20. R.V. Cadman et al., Phys. Rev. Lett. 86, 967 (2001).

21. K. Suda et al., Nucl. Instrum. Methods A 572, 745 (2007) doi: 10.1016/j.nima.2006.11.040.

22. H. Okamura et al., AIP Conf. Proc. 293, 84 (1994).
23. G.G. Ohlsen, Rep. Prog. Phys. 35, 717 (1972).

24. H. Okamura et al., Phys. Rev. C 66, 054602 (2002).

25. J.A. Kuehner et al., Phys. Rev. Lett. 35, 7 (1975).

26. T. Ichihara et al., Nucl. Phys. A 569, 287c (1997).

27. R.B. Wiringa et al., Phys. Rev. C 51, 38 (1995).

28. R. Machleidt et al., Phys. Rev. C 63, 024001 (2001).

29. V.G.J. Stoks et al., Phys. Rev. C 49, 2950 (1994).

30. A. Deltuva et al., Phys. Rev. C 71, 054005 (2005).

31. A. Deltuva et al., Phys. Rev. C 72, 054004 (2005).

32. P.F. Bedaque, U. van Kolck, Annu. Rev. Nucl. Sci. 52, 339 (2002).

33. E. Epelbaum, Prog. Part. Nucl. Phys. 57, 654 (2006). 